\title{
MicroRNA bta-miR-365-3p inhibits proliferation but promotes differentiation of primary bovine myoblasts by targeting the activin $A$ receptor type I
}

Dan Hao ${ }^{1,2}$, Xiaogang Wang ${ }^{1}$, Xiao Wang ${ }^{3}$, Bo Thomsen ${ }^{2}$, Yu Yang ${ }^{1}$, Xianyong Lan ${ }^{1}$, Yongzhen Huang ${ }^{1}$ and Hong Chen ${ }^{1 *}$

\begin{abstract}
Background: MicroRNAs act as post-transcriptional regulators that repress translation or degrade mRNA transcripts. Each microRNA has many mRNA targets and each mRNA may be targeted by several microRNAs. Skeletal muscles express a plethora of microRNA genes that regulate muscle development and function by controlling the expression of protein-coding target genes. To expand our understanding of the role of microRNA, specifically btamiR-365-3p, in muscle biology, we investigated its functions in regulating primary bovine myoblast proliferation and differentiation.

Results: Firstly, we found that bta-miR-365-3p was predominantly expressed in skeletal muscle and heart tissue in Chinese Qinchuan beef cattle. Quantitative PCR and western blotting results showed that overexpression of btamiR-365-3p significantly reduced the expression levels of cyclin D1 (CCND1), cyclin dependent kinase 2 (CDK2) and proliferating cell nuclear antigen (PCNA) but stimulated the expression levels of muscle differentiation markers, i.e., MYOD1, MYOG at both mRNA and protein level. Moreover, downregulation of bta-miR-365-3p increased the expression of CCND1, CDK2 and PCNA but decreased the expression of MYOD1 and MYOG at both mRNA and protein levels. Furthermore, flow cytometry, EdU proliferation assays and immunostaining results showed that increased levels of bta-miR-365-3p suppressed cell proliferation but promoted myotube formation, whereas decreased levels of bta-miR-365-3p resulted in the opposite consequences. Finally, we identified that activin A receptor type I (ACVR1) could be a direct target of bta-miR-365-3p. It was demonstrated that bta-miR-365-3p can bind to the $3^{\prime} U T R$ of ACVR1 gene to regulate its expression based on dual luciferase gene reporter assays. Consistently, knock-down of ACVR1 was associated with decreased expressions of CDK2, CCND1 and PCNA but increased expression of MYOG and MYOD1 both at mRNA and protein level.
\end{abstract}

Conclusion: Collectively, these data suggested that bta-miR-365-3p represses proliferation but promotes differentiation of bovine myoblasts through several biological mechanisms involving downregulation of ACVR1.

Keywords: ACVR1, Bta-miR-365-3p, Cattle, Primary bovine myoblast

\footnotetext{
* Correspondence: chenhong1212@263.net

'College of Animal Science and Technology, Northwest A\&F University,

Shaanxi Key Laboratory of Animal Genetics, Breeding and Reproduction, Yangling 712100, Shaanxi, China

Full list of author information is available at the end of the article
}

(c) The Author(s). 2021 Open Access This article is licensed under a Creative Commons Attribution 4.0 International License, which permits use, sharing, adaptation, distribution and reproduction in any medium or format, as long as you give appropriate credit to the original author(s) and the source, provide a link to the Creative Commons licence, and indicate if changes were made. The images or other third party material in this article are included in the article's Creative Commons licence, unless indicated otherwise in a credit line to the material. If material is not included in the article's Creative Commons licence and your intended use is not permitted by statutory regulation or exceeds the permitted use, you will need to obtain permission directly from the copyright holder. To view a copy of this licence, visit http://creativecommons.org/licenses/by/4.0/. The Creative Commons Public Domain Dedication waiver (http://creativecommons.org/publicdomain/zero/1.0/) applies to the data made available in this article, unless otherwise stated in a credit line to the data. 


\section{Background}

Skeletal muscles originate from embryonic structures called somites, where mononuclear myoblasts proliferate, differentiate and fuse to multinucleated myotubes and subsequently differentiate into myofibers [1]. Myofibers vary with respect to their myosin heavy chain isoforms (fast versus slow) and types of energy metabolism (oxidative versus glycolytic). The number of myofibers is constant, but myofibers can increase in size by fusion with muscle stem cells, the satellite cells [2]. For example, adult skeletal muscle has a remarkable ability to repair after injury, leading to new myofiber formation in this process that involves satellite cells [3]. In addition, skeletal muscle mass and muscle fiber characteristics play key roles in the determination of meat yield and quality in cattle. Therefore, understanding the molecular processes and genetic networks underlying myogenesis and muscle development will provide fundamental information for cattle breeding programs.

The mature microRNAs (miRNAs) are small RNA molecules ( 22 nucleotides), which have been widely identified in humans and animals since they were firstly discovered in the nematode Caenorhabditis elegans [4]. MicroRNAs act as post-transcriptional regulators that repress translation or degrade mRNA transcripts through either complete (canonical sites) or incomplete (non-canonical sites) complementarity with the 3'UTR of target mRNAs. Currently, the sequencing technologies are accelerating the discovery of microRNAs. Meanwhile, effective microRNA target sites are accurately predicted using various computational approaches [5]. The latest database of miRbase (Release 22.1) contains 48,860 distinct mature miRNA (miR) sequences from 271 organisms, including 1143 miRNAs from cattle [6].

Previous studies have revealed that tissue-specific and developmental stage-specific miRNAs play critical functional roles in diverse cellular, physiological and developmental processes [7-9]. For example, muscle-specific miRNAs such as miR-1, miR-133 and miR-206 participates in ontogenesis and skeletal myogenesis through a modulation of muscle differentiation genes [10-12]. Profiling of miRNA expression patterns among eleven different tissues from beef cattle showed that bta-miR365-3p was ubiquitously expressed but with the highest expression level in muscle, which suggested its regulatory role in muscle tissue [13]. Furthermore, bta-miR365-3p was differentially expressed between fast- and slow-type muscles (semitendinosus versus masseter) in Japanese Black steers [14]. Moreover, 2.6 fold higher expression of bta-miR-365-3p was found in the adult stage compared to the fetal stage of muscle tissues in Qinchuan cattle. A similar tendency has been observed with well-known muscle-specific miRNA bta-miR-1, but opposite with bta-miR-206 [15] (Fig. S1A). Remarkable, the total number of sequence reads of bta-miR-365-3p in the proliferation stages were almost 3.5 times higher than in the differentiation stages of skeletal musclederived satellite cells in Chinese Simmental calves. Similar results were also found in bta-miR-378a-3p and bta-miR-23a [16].

Based on this, we speculate that bta-miR-365-3p probably plays an important role in muscle tissue development. Notably, it has been reported that miR-365-3p negatively regulated histone deacetylase 4 (HDAC4) to stimulate primary chondrocyte proliferation and differentiation in mouse and chicken [17] and to contribute to osteoarthritis pathogenesis in humans [18]. However, few studies reported the characterizations of the target genes and the regulatory network of bta-miR-365-3p in muscle cells. Thus, the objective of our study is to assess the expression level of bta-miR-365-3p in various bovine tissues and to investigate its influences on proliferation and differentiation of primary myoblast.

\section{Materials and methods \\ Animal and cell culture}

All animal experiments were approved by the Animal Care Commission of the College of Veterinary Medicine of Northwest A\&F University (Permit Number: NWAF AC1019). Six tissue samples, i.e., heart, liver, spleen, lung, kidney, longissimus dorsi muscle, were collected from sixty-day-old fetuses $(n=3)$ and two-year-old adults $(n=3)$ of the Chinese Qinchuan (QC) beef cattle breed. All samples were provided by Shanxi Kingbull Livestock Co., Ltd., Baoji city, China. Primary bovine myoblasts (PBMs) were isolated from fetal longissimus dorsi muscle following the established protocols [19]. Myoblasts were cultured in growth medium (GM) consisting of high-glucose Dulbecco's modified Eagle's medium (DMEM, Gibco) with $1 \%$ penicillinstreptomycin (HyClone) and $20 \%$ fetal bovine serum (TransGen, Beijing, China). Myoblast differentiation was stimulated in DMEM containing $2 \%$ horse serum (HyClone) and 1\% penicillin-streptomycin (differentiation medium, DM). Cells were incubated at $37^{\circ} \mathrm{C}$ with $5 \% \mathrm{CO}_{2}$.

\section{Plasmid construction and transfection}

A DNA fragment containing the precursor sequence of bta-miR-365-3p was obtained from genomic DNA of QC cattle by PCR and was inserted into the pcDNA3.1(+) vector using T4 DNA ligase (Takara, Dalian, China). The resulting plasmid was named OPmiR-365$3 p$ and used for overexpression of bta-miR-365-3p in PBMs.

The sequence of bta-miR-365-3p inhibitor is AUAAGGAUUUUUAGGGGCAUUA. With a 21-23 nt 2 '-methoxy modified RNA oligonucleotide design, the 
bta-miR-365-3p inhibitor is a purified molecules that specifically and effectively inhibit endogenous mature bta-miR-365-3p's activities. The sequence of the inhibitor's negative control is CAGUACUUUUGUGU AGUACAA, which acted as the control group for the bta-miR-365-3p inhibitor treatment group (Table S1).

A DNA fragment containing the target site of btamiR-365-3p in the 3'UTR of bovine AVCR1 was amplified by PCR and cloned into the XhoI and NotI sites of the psiCHECK-2 dual-luciferase reporter vector (Promega, Madison, WI, USA) and the construct was named ACVR1-wild. Mutagenic primers were used to mutagenize the bta-miR-365-3p target site, which was cloned into psiCHECK-2 to create ACVR1-mutant. Three siRNAs of $A C V R 1$ were used to inhibit the expression of ACVR1 in PBMs, including siACVR1-1, siACVR1-2 and siACVR1-3. The sequence of the siACVR1s is shown in Table S1.

Cells were transfected with OPmiR-365-3p, the inhibitor of bta-miR-365-3p, inhibitor N. C, ACVR1-wild, ACVR1-mutant and siACVR1s using Lipofectamine 2000 (Invitrogen, Grand Island, NY, USA) and incubated with $5 \% \mathrm{CO}_{2}$ at $37^{\circ} \mathrm{C}$. The inhibitors and siRNAs were purchased from GenePharma (Shanghai, China). All experiments were performed in triplicate. All primers, inhibitors and the siRNAs sequences are listed in Table S1.

\section{RNA extraction and qRT-PCR}

Total RNA was extracted from six different tissues and from PBMs using TRIzol reagent (Takara, Japan). After assessing RNA purity and concentration by spectrophotometry using a NanoDrop 2000 (Wilmington, USA) and $0.8 \%$ agarose gel electrophoresis, $1000 \mathrm{ng}$ RNAs were transcribed into complementary DNA (cDNA) with PrimeScript RT reagent kit for use in qRT-PCR with SYBR Green Master Mix Reagen kit (GenStar, Beijing, China). The specific stem-loop of bta-miR-365-3p was used to synthesize the first cDNA. All primers are listed in Table S1. The method of $2^{-\Delta \Delta \mathrm{Ct}}$ was used to calculate the relative expression levels.

\section{Western blot analysis}

All proteins were extracted from PBMs at $4{ }^{\circ} \mathrm{C}$ using the radioimmunoprecipitation assay lysis buffer (RIPA buffer) and phenylmethylsulfnoyl fluoride (PMSF) (Solarbio, Beijing, China). Proteins were measured and adjusted by using the BCA protein assay kit (MULTI SCIENCE, China) and denatured with $5 \times$ SDS loading buffer (Beyotime) at $98^{\circ} \mathrm{C}$ for $10 \mathrm{~min}$. The prepared proteins were separated by SDS-polyacrylamide gel electrophoresis and then transferred to polyvinylidene fluoride membranes. After being blocked with 5\% skim milk solution, membranes were incubated with the specific primary antibodies and the secondary antibody. We visualized the membranes using ChemiDoc ${ }^{\mathrm{Tn}} \mathrm{XRS}+$ system (BioRad Laboratories) and ECL Plus reagents (Solarbio, Beijing, China). The primary antibodies including antiCDK2 and anti-PCNA were obtained from Sangon Biotech (Shanghai, China). Here, anti-ACVR1, anti-cyclin D1, anti-MyoD and anti-MyoG were purchased from Abcam (Cambrige, MA, USA). Anti- $\beta$-actin were purchased from SungenBio (Tianjin, China). HRPconjugated Goat Anti-Rabbit IgG was obtained from BBI Life Science (Shanghai, China). All the primary antibodies were diluted with primary antibody dilution buffer that was obtained from Beyotime (Haimen, China). Image $\mathrm{Lab}^{\text {Tu }}$ Software 6.0.1 was used to calculate the grayscale value of the proteins.

\section{EdU and flow cytometry assay}

After the transfection of PBMs with the expression vectors, inhibitor and siRNAs, we employed the EdU proliferation assay to measure their influences on DNA synthesis using the Cell Light EdU DNA cell proliferation kit according to the instructions (RiboBio, Guangzhou, China). The stained cells were detected and calculated by fluorescence microscopy (DM5000B, Leica Microsystems). Cell cycle phases were assessed by a cell cycle testing kit (Multisciences, Hangzhou, China) on a flow cytometry instrument (FACS Canto II, BD Biosciences, USA). Briefly, the cells were seeded in 6-well plates and transfected for $24 \mathrm{~h}$ after the cells reached $60 \%$ confluence. Cold $70 \%$ ethanol was used to fix the harvested cells. After staining with $100 \mu \mathrm{g} / \mathrm{mL}$ of the PI master mix at $37^{\circ} \mathrm{C}$ for $30 \mathrm{~min}$, the cell suspension was subjected to flow cytometry.

\section{Immunofluorescence staining}

After inducing PBMs differentiation for $4 \mathrm{~d}, 4 \%$ paraformaldehyde in PBS was used to fix differentiated myoblast in a plate for $20 \mathrm{~min}$. $0.5 \%$ of Triton-X-100 was added to permeabilize the fixed myoblast for $10 \mathrm{~min}$ and the cells were blocked with $5 \%$ bovine serum albumin solution (BSA) at $4{ }^{\circ} \mathrm{C}$ for $2 \mathrm{~h}$. Subsequently, we incubated primary antibody (anti-MyHC diluted 1:250; Abcam, Cambridge, MA, USA) at $4{ }^{\circ} \mathrm{C}$ overnight and incubated the corresponding fluorescent secondary antibody at $4{ }^{\circ} \mathrm{C}$ for $2.5 \mathrm{~h}$. Finally, the cell nuclei were stained with DAPI and the images were captured by fluorescence microscope (DM5000B, Leica Microsystems, Germany). The degree of differentiation was measured by the fusion index that was calculated as the number of nuclei in the myotube and as a percentage of the total nuclei. 


\section{Dual-luciferase reporter assay}

Dual-luciferase reporter assay was applied to test the interaction of bta-miR-365-3p with its predicted targets. HEK293T cells were co-transfected with OPmiR-365-3p vector (or the empty vector) and other vectors containing ACVR1-wild or ACVR1-mutant. The dual-luciferase activity was analyzed on an MPPC luminescence analyzer (HAMAMATSU, Beijing, China) using the luciferase reporter assay kit (Promega, Madison, WI, USA) according to the manufacturer's instructions. The results were calculated as the ratios of firefly to Renilla luciferase activities in three independent replicates.

\section{Bioinformatics analysis}

The online databases TargetScan (http://www.targetscan.org/vert_72/) and miRmap (https://mirmap.ezlab. org/) were used to search for the targets of bta-miR-3653p [20, 21]. VENNY tool (version 2.1) (https://bioinfogp. cnb.csic.es/tools/venny/index.html) was used to obtain the common targets from the two databases [22]. The $\mathrm{R}$ package clusterProfiler [23] was used to cluster the enrichments of Gene Ontology (GO) and Kyoto Encyclopaedia of Genes and Genomes (KEGG) for the common genes.

\section{Statistical analysis}

All the quantitative data were presented as the mean \pm SD. Each group has three independent experiments. Student' $t$-test procedure was used to analyze the statistical significance between groups were analyzed by SPSS v19.0. In this study, one asterisk, two asterisks and three asterisks indicated $P<0.05, P<0.01$ and $P<0.001$ between groups, respectively.

\section{Results}

\section{Bta-miR-365-3p expression in cattle tissue and PBMs}

In order to investigate the functional roles of btamiR-365-3p, we firstly detected the expression levels of bta-miR-365-3p in six different tissues of two developmental stages in $\mathrm{QC}$ cattle using quantitative PCR. The results showed that muscle and spleen tissues had the higher expression level in the fetus, while the expression of bta-miR-365-3p was highest in heart tissue of adult stage (Fig. 1a). Additionally, the expression levels of bta-miR-365-3p were significantly different $(P$-value $<0.05)$ between adult and fetal stages in the liver, heart and muscle tissues (Fig. 1a). We also found that the expression levels of bta-miR-365-3p exhibited a slightly decreased trend during PBM proliferation (Fig. 1b), while a dynamic expression profile that peaked on day four was observed during differentiation and subsequently rapidly decreased on day 6 (Fig. 1c). In the cultured myoblast cells transfected with the expression vector OPmiR- 365-3p, quantitative PCR results showed that btamiR-365-3p was significantly overexpressed, whereas the expression levels of bta-miR-2333 and bta-miR193a that map close to bta-miR-365-3p on Bos taurus autosome (BTA) 19 were not significantly overexpressed (Fig. 1d and e), which indicated that the expression vector was constructed successfully.

\section{Bta-miR-365-3p suppresses PBM proliferation}

The proteins encoded by CDK2 (cyclin-dependent kinase 2), PCNA (proliferating cell nuclear antigen) and CCND1 (cyclin D1) have been identified to perform critical functions in $G_{1}, S$ and $G_{2}$ phases during cell cycle progression [24-26]. The results of qRT-PCR and western blotting showed that the expressions of CCND1, $C D K 2$ and $P C N A$ were significantly decreased at both the mRNA and protein levels after transfecting PBMs with OPmiR-365-3p (Fig. 2a). Flow cytometer assays showed that PBM numbers were lower in the $\mathrm{G}_{2}$-phase $(12.78 \%)$ and in the $S$-phase $(16.91 \%)(P<0.05$ and $P=$ 0.08 , respectively), whereas the proportion of PBMs was increased in the $G_{0} / G_{1}$-phase, when bta-miR-365-3p was overexpressed (Fig. 2b, c and d). The EdU proliferation assays revealed a $36.86 \%$ reduction in mitotic activity of PMBs after transfection with OPmiR-365-3p $(P<0.01)$ (Fig. 2e and f). However, inhibition of bta-miR-365-3p significantly increased the expressions of the proliferation marker genes CCND1, CDK2 and PCNA at both mRNA and protein levels (Fig. 3a). The proportion of PBMs was increased $20.06 \%$ in $S$-phase $(P<0.05)$, and decreased $3.1 \%$ in $G_{0} / G_{1}$-phase after we knocked down bta-miR-365-3p in PBMs (Fig. 3b, c and d). In addition, the number of EdU-positive cells increased $15.4 \%$ in the bta-miR-365-3p inhibitor group (Fig. 3e and f). Therefore, we suggested that the overexpression of bta-miR365-3p suppressed PBM proliferation, while knockdown of bta-miR-365-3p promoted PBM proliferation.

\section{Bta-miR-365-3p promotes PBMs differentiation}

To understand the function of bta-miR-365-3p for PBMs differentiation, we monitored its expression levels following induction of differentiation and overexpression by continuously transfecting with OPmiR-365-3p. Expectedly, it also showed a peak expression level of btamiR-365-3p during differentiation on day 4 (Fig. 4a). Subsequently, the effect of bta-miR-365-3p on PBM differentiation was assessed by an approximately 10-fold overexpressing after the transfection with OPmiR-3653p (Fig. 4b), or 5-fold reduction of expression levels using an inhibitor of bta-miR-365-3p on differentiation day 4 (Fig. 4c). Both mRNA and protein expression levels of two muscle differentiation marker genes (i.e., MYOD1 and MYOG) increased by OPmiR-365-3p (Fig. 4d and e), but decreased by bta-miR-365-3p 
(A)

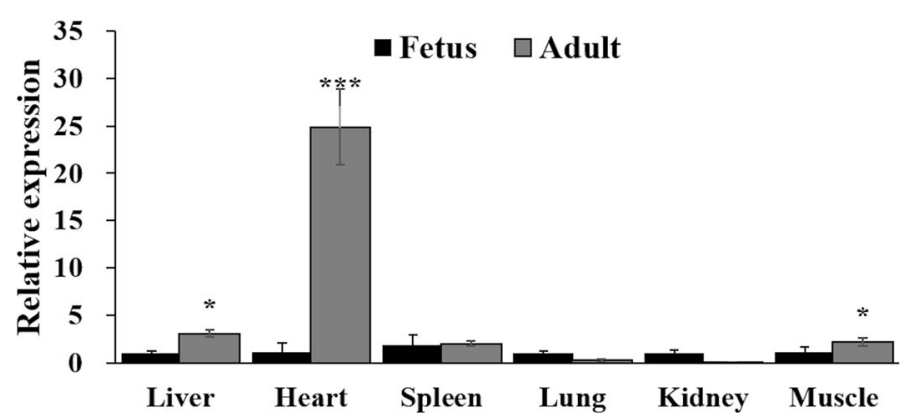

(C)

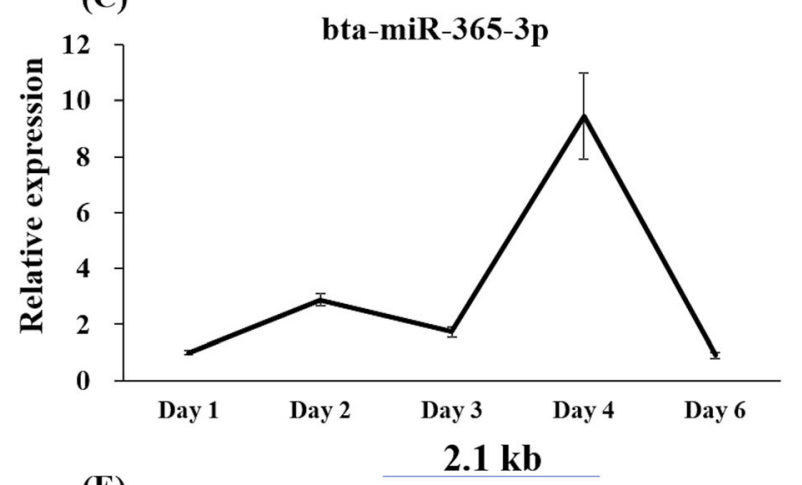

(B)

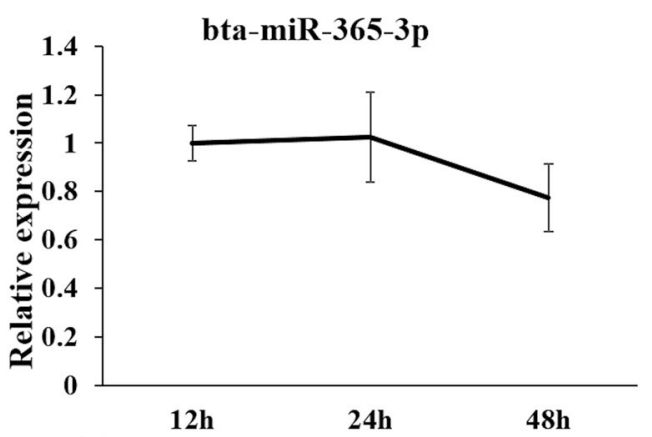

(D)

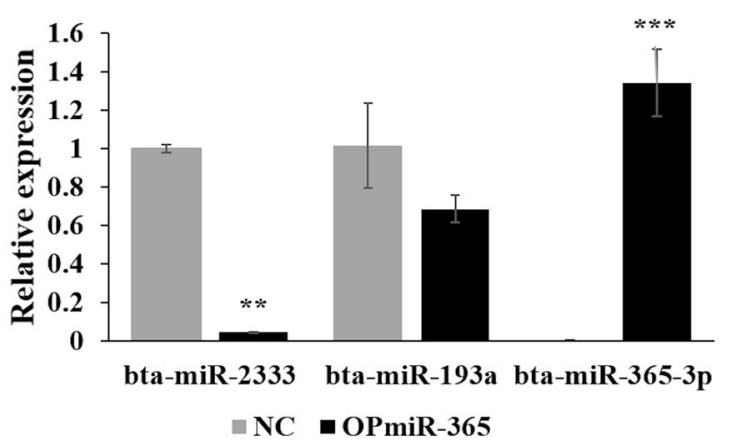

$10.6 \mathrm{~kb}$

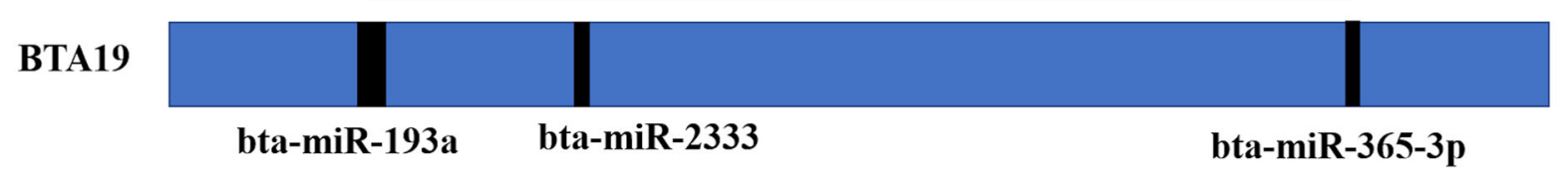

Fig. 1 The expression level of bta-miR-365-3p at different development stages of cattle tissues and PBMs. a Differential expression level of btamiR-365-3p in different tissues between fetal stage and adult stage of QC cattle. b bta-miR-365-3p expression levels during PBMs proliferation. $\mathbf{c}$ bta-miR-365-3p expression levels during PBMs differentiation with no treatment. $\mathbf{d}$ The expression levels of bta-miR-2333, bta-miR-193a and btamiR-365-3p in PBMs, when transfected with OPmiR-365-3p in PBMs for $24 \mathrm{~h}$ in proliferation stage. NC, negative control with transfecting empty plasmid pcDNA 3.1. Note: The expression level of bta-miR-365-3p showed in the histogram was 100 times smaller. e The location of bta-miR-365$3 p$ and its neighbor miRNAs in the bovine chromosome BTA19. All data are shown as mean \pm SD for three biological replicates, the error bars indicated the SD among three repeats. Note:PBMs: primary bovine myoblasts; OPmiR-365-3p: the constructed pcDNA3.1 vector overexpressing bta-miR-365-3p; SD: standard deviation. ${ }^{*} P<0.05,{ }^{* *} P<0.01,{ }^{* *} P<0.001$

inhibitor (Fig. 4f and g). Immunofluorescence staining results showed that bta-miR-365-3p overexpression gave a higher amount of MyHC-positive myotubes compared to the control group (Fig. 4h and j), while the opposite result was observed in the treatment with the bta-miR365-3p inhibitor (Fig. 4i and j). Therefore, we suggested that bta-miR-365-3p promoted PBM differentiation.

\section{ACVR1 is a target gene of the bta-miR-365-3p}

In silico prediction, TargetScan and miRmap revealed 1354 and 354 putative target genes of bta-miR-365-3p, respectively. The intersection of the predicted targets gave 101 genes, which were used in a KEGG pathway analysis (Fig. 5a). The five most significant signaling pathways (adjusted $P$-value $<0.05$ ) were parathyroid hormone synthesis, secretion and action (bta04928), endocytosis (bta04144), estrogen signaling pathway (bta04915), phospholipase D signaling pathway (bta04072) and choline metabolism in cancer (bta05231) (Fig. 5b). Interestingly, the putative target genes of btamiR-365-3p, ACVR2A (activin A receptor type 2A), SP1 (Sp1 transcription factor) and ACVR1 (activin A receptor type 1) were enriched in TGF-beta signaling pathway (bta04350) ( $P$-value <0.1) (Table S2). Moreover, the presence of target sites in the 3' UTR of these three genes were analyzed in fourteen different animal species, which showed a strong conservation of the bta-miR-365$3 \mathrm{p}$ target sites, especially in ACVR1 (Fig. 5c). The Fig. 5d showed that the expression level of $A C V R 1$ was inhibited when the endogenous bta-miR-365-3p was highly expressed on day 2 and especially on day 4 . By contrast, the expression level of $A C V R 1$ showed higher expression 


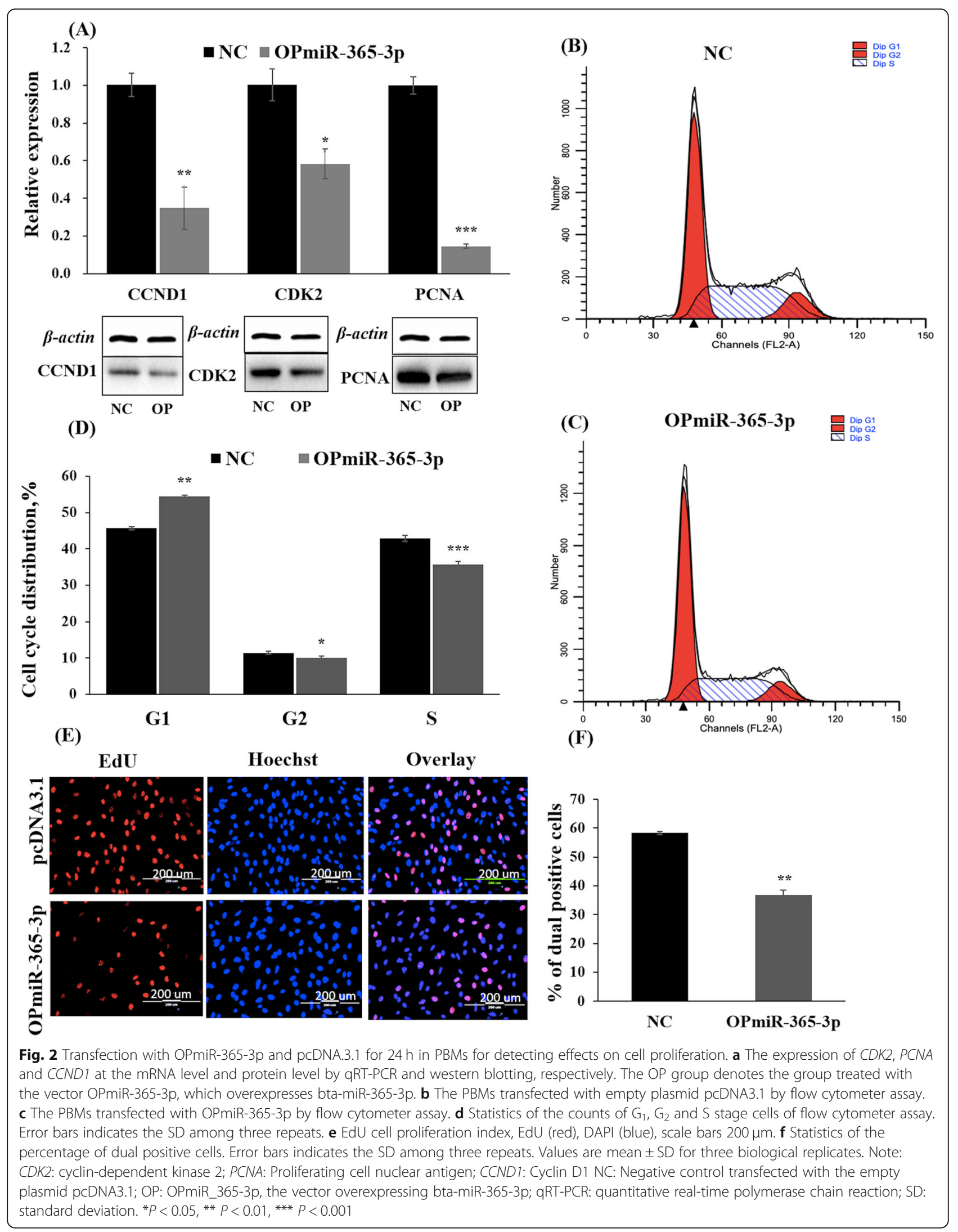




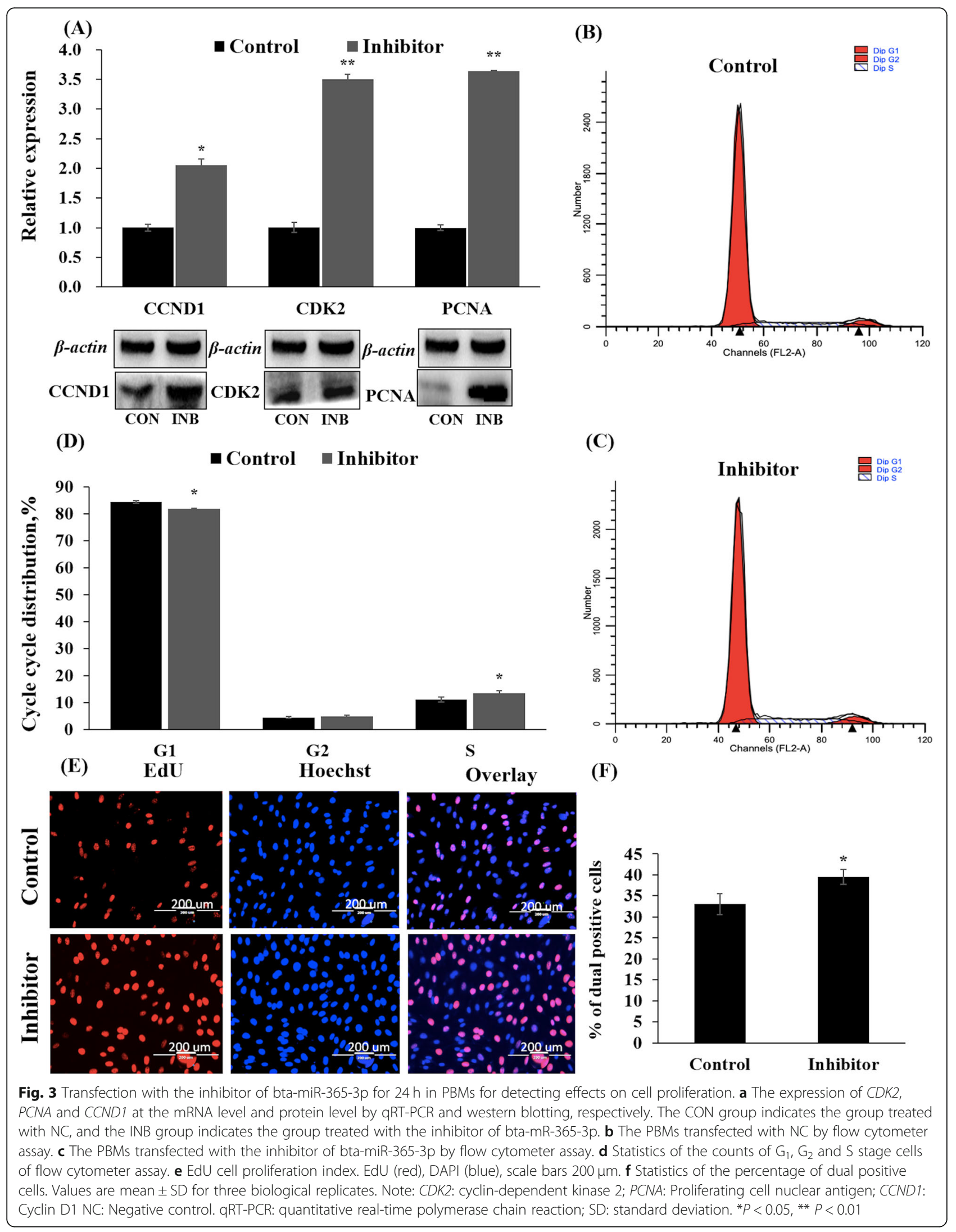






when the endogenous bta-miR-365-3p was downregulated on day 3 (Fig. 5d). Furthermore, expression of $A C V R 1$ was significantly downregulated on day 4 after transfection with OPmiR-365-3p in PBMs (Fig. 5e). Consistently, overexpression of bta-miR-365-3p negatively regulated the ACVR1 mRNA and protein levels in PBMs on day 4 (Fig. 5e and Fig. 5f). We also conducted a dualluciferase activity experiment to test the interaction of bta-miR-365-3p with the 3'UTR of ACVR1. The data showed that the luciferase activity was significantly decreased when co-transfecting the OPmiR-365-3p and ACVR-wild vectors in PBMs. In contrast, the luciferase activity was unaffected in co-transfections of OPmiR365-3p and ACVR1-mutant. Collectively, these results suggested that $A C V R 1$ could be a direct target gene for bta-miR-365-3p (Fig. 5g).

\section{siACVR1 inhibited PBM proliferation but promoted PBM differentiation}

Next, we employed siRNA technology to address the role of $A C V R 1$ in PBM. Three different siRNAs were designed to target the bovine $A C V R 1$ and to be transfected in PBMs. It showed that siACVR1-1 downregulated the expression of $A C V R 1$ efficiently at both the mRNA and 
(A)

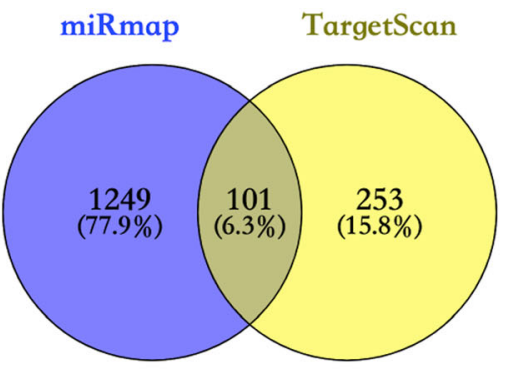

(C)

\begin{tabular}{ll|l|l|}
\hline Species & 3'UTR of ACVR1 & 3'UTR of ACVR2A & 3'UTR of SP1 \\
\hline Cow & UGGGCAUUA & GGGCAU---U & GGGCAUUA \\
\hline Mouse & UGGGCAUUA & GGGCAU---U & GGGCAUUA \\
\hline Rabbit & UGGGCAUUA & GGGCA---UU & GGGCAUUA \\
\hline Pig & UGGGCAUUA & GGGCAUU & GGGCAUUA \\
\hline Cat & UGGGCAUUA & GGGCAU---U & GGGCAUUA \\
\hline Dog & UGGGCAUUA & GGGCAU---U & GGGCAUUA \\
\hline Elephant & UGGGCAUUA & GGGCAU---U & GGGCAUUA \\
\hline Rat & UGGGCAUUA & GGGCAU---U & $\otimes$ \\
\hline Opossum & UGGGCAUUA & GGGCA---UU & $\otimes$ \\
\hline Brown bat & UGGGCAUUA & GGGCAU---U & $\otimes$ \\
Human & UGGGCAUUA & $\otimes$ & $\otimes$ \\
\hline Chimp & UGGGCAUUA & $\bigotimes$ & $\otimes$ \\
\hline Rhesus & UGGGCAUUA & $\otimes$ & $\otimes$ \\
Macaw & UGGGCAUUA & $\bigotimes$ & $\otimes$ \\
\hline
\end{tabular}

(D)

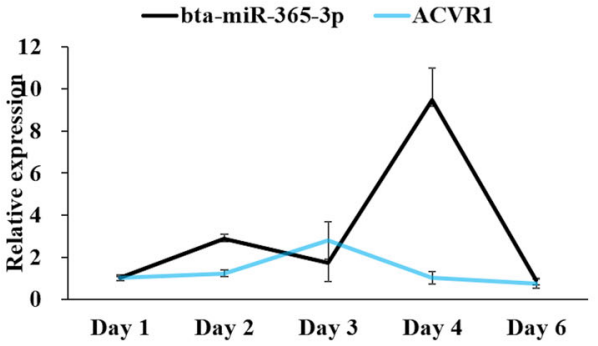

(F)

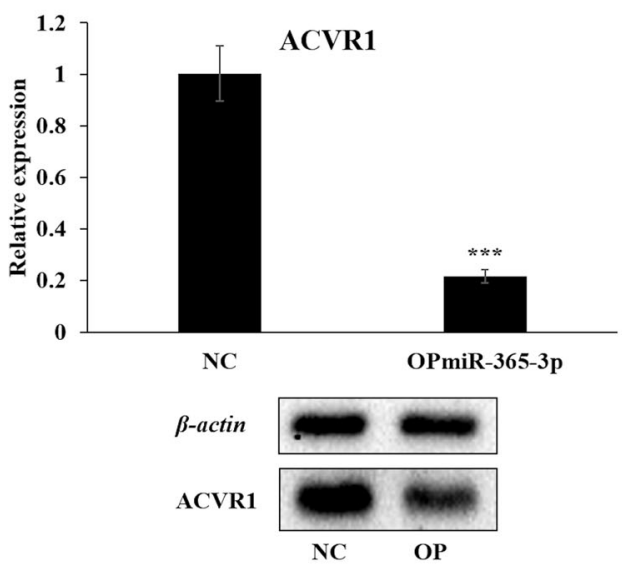

(G)
(B)

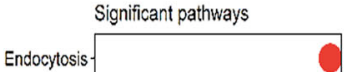

Parathyroid hormone synthesis, secretion and action

Estrogen signaling pathway

Phospholipase $\mathrm{D}$ signaling pathway

MAPK signaling pathway

Choline metabolism in cancer

mTOR signaling pathway

Cushing syndrome

Rap1 signaling pathway

CAMP signaling pathway

Human cytomegalovirus infection-

Gap junction -

TGF-beta signaling pathway -

Endocrine resistance -

Cholinergic synapse

Relaxin signaling pathway

FoxO signaling pathway

Signaling pathways regulating pluripotency of stem cells.

Autophagy - animal -

Hedgehog signaling pathway-

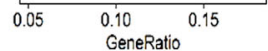

(E)
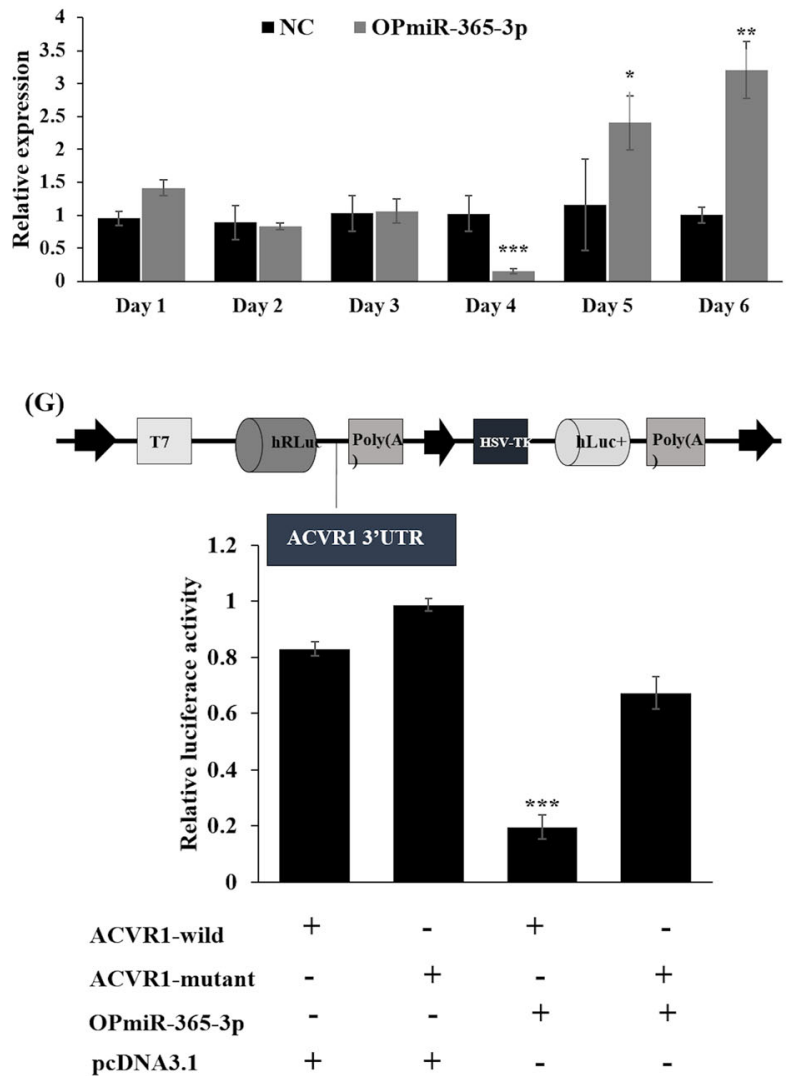

Fig. 5 (See legend on next page.) 
(See figure on previous page.)

Fig. 5 Bovine ACVR1 is a target gene for miR-365-3p. a The common target genes of bta-miR-365-3p from two databases, miRmap and TargetScan. b The KEGG pathways associated with the common target genes of bta-miR-365-3p. The red to blue color indicated that p.adjust values was increasing, and the red circle means the genes were enriched in the significant pathway; the size of the circle indicates the gene counts in the pathway. $\mathbf{c}$ Conservation among various species of the bta-miR-365-3p target sequences in the 3'UTR of ACVR1, ACVR2A, SP1. d The expression level of ACVR1 during PBMs differentiation. e The expression level of ACVR1 after transfection with OPmiR-365-3p for continuously inducing differentiation 6 days. $\mathbf{f}$ The mRNA and protein expression of ACVR1 when transfected with the interference siACVR1-1 cultured with growth medium (GM) for proliferating $24 \mathrm{~h} . \mathbf{g}$ The structure of the psiCHECK-2 dual-luciferase reporter vector, and the luciferase activity test when transfected with ACVR1- wild, ACVR1-mutant, OPmiR-365-3p and pCDNA3.1(NC). Note: ACVR1: activin A receptor type l; ACVR2A: activin A receptor type 2A; Sp1: transcription factor; ACVR1- wild: psiCHECK-2 dual-luciferase reporter vector of the ACVR1 3'UTR region. ACVR1-mutant: psiCHECK-2 dual-luciferase reporter vector of the ACVR1 3'UTR region with mutation; SD: standard deviation. ${ }^{*} P<0.05$, ${ }^{* *} P<0.01,{ }^{* * *} P<0.001$

protein levels in PBMs (Fig. 6a). Furthermore, the analysis of q-RT-PCR and western blotting found that siACVR1-1 also significantly decreased CDK2 expression at both mRNA and protein levels. CCND1 and PCNA were only slightly decreased at mRNA level but significantly reduced at the protein level (Fig. 6b). In the EdU proliferation assay, the percentage of dual positive PBMs was significantly lower in cells after knock-down of ACVR1 than in non-treated cells (Fig. 6c and Fig. 6d). Conversely, the muscle differentiation markers $M Y O D 1$ and $M Y O G$ were increased at both mRNA and protein levels after knock-down of ACVR1 in PBMs (Fig. 6e and f). Therefore, overexpression of bta-miR-365-3p and knock-down of $A C V R 1$ expression have similar consequences on PBMs proliferation and differentiation.

\section{Discussion}

\section{miRNAs in bovine skeletal muscle}

Skeletal muscle mass and muscle fiber characteristics are highly related to important economical traits such as meat quality and yield in beef cattle [27]. Understanding the molecular genetics of bovine skeletal muscle development will, therefore, provide important information for using in cattle breeding programs. Recently, advanced sequencing and bioinformatics technologies as well as the annotated databases like miRbase have revealed several miRNAs associated with bovine skeletal muscle development and differentiation of satellite cells $[6,13-16,28]$. MiRNAs regulate protein synthesis by targeting mRNAs; so far four microRNAs modulating bovine skeletal muscle development and function have been deposited in miRTarBase, which provides information about experimentally validated miRNA-target interactions [29]. Six miRNAs regulating proliferation, apoptosis and differentiation of PBMs through targeting of various functional genes have been confirmed by various experimental methods [30-35] (Table 1). For example, miR-744 was abundantly expressed in fetal stage of Qinchuan cattle and has been confirmed to positively regulate proliferation of skeletal muscle satellite cells $[15,31]$.

\section{The expression profile and functional roles of bta-miR- 365-3p}

Previous studies have shown that the expression levels of bta-miR-365-3p were significantly differently expressed in the fast- and slow-type skeletal muscles in the stages of myoblast differentiation and the different developmental stages of cattle [13-16], supporting its functional roles in skeletal muscle development. Skeletal muscle development can be divided into the prenatal stage that decides the muscle fibers numbers and the postnatal stage that mainly generates the muscle fiber size [36]. Firstly, we demonstrated that bta-miR-365-3p was highly expressed in heart and skeletal muscle tissues in Qinchuan cattle (Fig. 1a), which was consistent with the previous transcriptome study in various tissues of Angus crossbred cattle [13]. Subsequently, bta-miR-365$3 p$ had higher expression in adult than in fetal stages of muscle tissues that was similar to bta-miR-1 in Sun et al. [15] (Fig. S1A). Thus, the results potentially indicate more important roles of bta-miR-365-3p in the postnatal stage development of skeletal muscle to affect the fiber size than in the prenatal stage development to affect the muscle fibers numbers.

The dynamic process of myoblast developed to myofiber involved proliferation, determination, differentiation and maturation phases [37, 38]. In our study, endogenous btamiR-365-3p showed higher expression in the maturation stage ( $72 \mathrm{~h}-96 \mathrm{~h}$, myotubes to form myofiber) than in the early differentiation stage $(24 \mathrm{~h}-72 \mathrm{~h}$, mononucleated fuse to multinucleated myotubes). The same expression tendency was found in the specific-related skeletal muscle development miRNAs, such as bta-miR-1 and bta-miR-23a but not bta-miR-125b in a previous study (Fig. S1B) [16]. In the early myoblast differentiation, bta-miR-1 expression was higher than bta-miR-365-3p, suggesting that bta-miR-1 is more important role for the early differentiation stage than bta-miR-365-3p. In agreement, we observed that the endogenous bta-miR-365-3p plays an important role in the maturation stage of the 


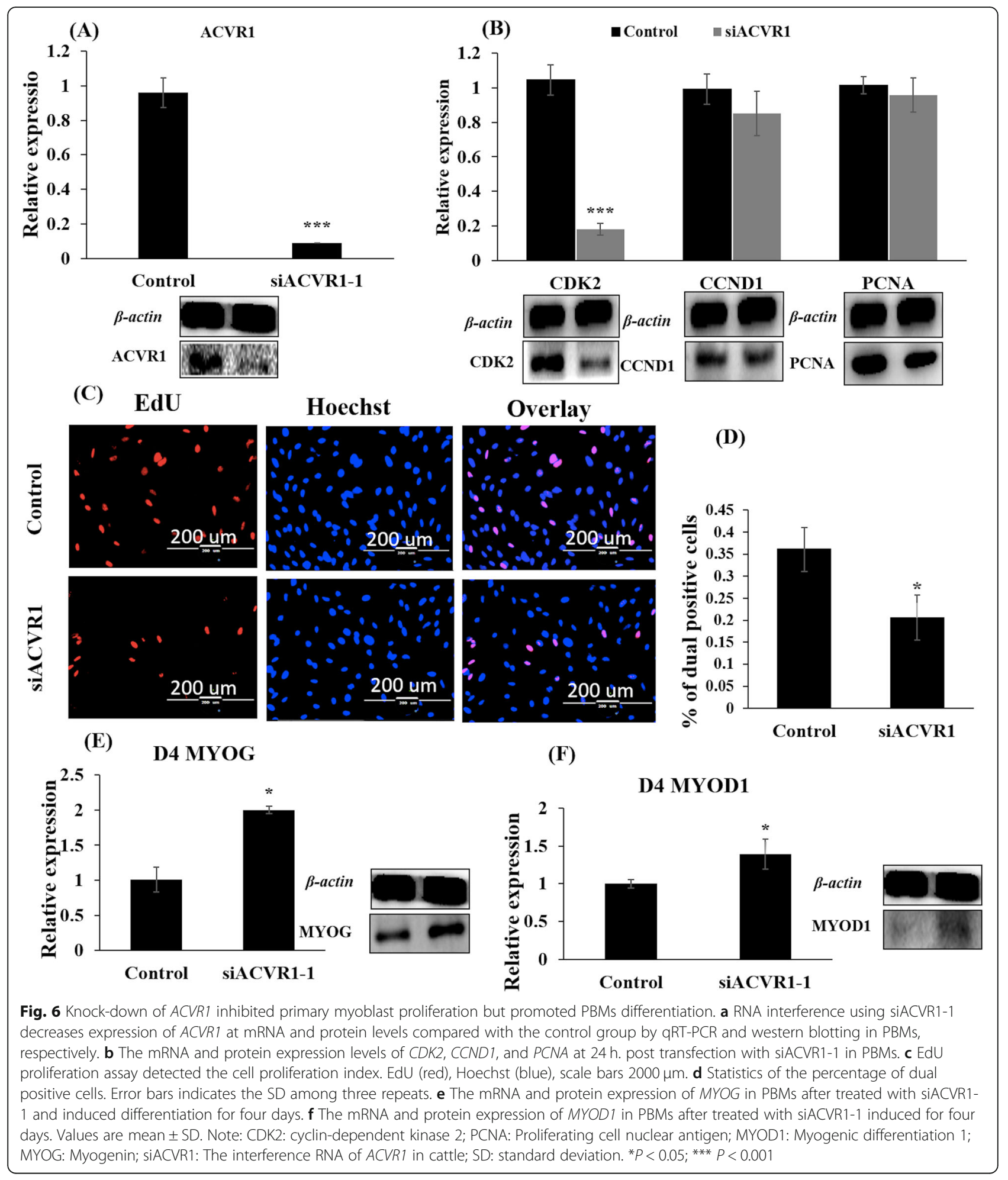

myoblast differentiation process of rather than in early myoblast differentiation stage [16] (Fig. 1c). The lower expressed after 4 days differentiation, indicated that once the myofibers were fused, the function of btamiR-365-3p may be reduced.
It has been reported that miR-365-3p served as a therapeutic biomarker for various cancers and tumors such as lung cancer [39, 40], colon cancer [28], pancreatic cancer [41], breast cancer [42] and gastric tumorigenesis [43]. Moreover, miR-365-3p inhibited vascular 
Table 1 The published miRNAs to skeletal muscle myoblast development in cattle

\begin{tabular}{lllll}
\hline miRNAs name & Target gene & Target ID & Associated phenotype & References PMID \\
\hline bta-miR-1 & HDAC4 & 517,559 & Skeletal muscle satellite cell myogenic differentiation & 26424132 \\
bta-miR-206 & HDAC4 & 517,559 & Skeletal muscle satellite cell myogenic differentiation & 26424132 \\
bta-miR-1 & LOC536229 & 536,229 & Skeletal muscle satellite cell myogenic differentiation & 26424132 \\
bta-miR-206 & LOC536229 & 536,229 & Skeletal muscle satellite cell myogenic differentiation & 26424132 \\
bta-miR-23a & ZNF423 & 508,025 & Adipogeneses in skeletal muscle & 28255176 \\
bta-miR-27b & MSTN & 281,187 & Skeletal muscle hypertrophy & 23510267 \\
bta-miR-208b & CDKN1A & 513,497 & Promoted skeletal muscle cell proliferation & 30317561 \\
bta-miR-744 & Wnt5a & 530,005 & Promoted skeletal muscle cell proliferation while inhibited & 31051333 \\
bta-miR-744 & CaMKII & $109,560,236$ & Promoted skeletal muscle cell proliferation while inhibited & 31051333 \\
bta-miR-148a-3p & KLF6 & 505,884 & the apoptosis and differentiation & Inhibited muscle cell proliferation but promoted apoptosis \\
bta-miR-378a-3p & HDAC4 & 517,559 & Promoted myoblast differentiation and inhibited proliferation & 276793769 \\
bta-miR-125b & IGF2 & 281,240 & Sponged by IncMD to promote myoblast differentiation & 27589905 \\
bta-miR-107 & Wnt3a & 522,467 & Suppress cell differentiation and did not affect cell proliferation & 29858062 \\
bta-miR-885 & MYOD1 & 281,938 & Promote proliferation but inhibit differentiation & 331985035 \\
\hline
\end{tabular}

smooth muscle cell proliferation through the targeting of CCND1 [44]. This agrees well with the present study, demonstrating that bta-miR-365-3p acted as a negative regulator of PBM proliferation. Thus, cell cycle analysis with high levels of bta-miR-365-3p showed an increased the percentage of cells in the $G_{0} / G_{1}$-phase and reduced the number of cells in S-phase, whereas, downregulated bta-miR-365-3p with its inhibitor showed opposite effects. Consistently, CDK2, CCND1 and PCNA were all shown to be downregulated when overexpressing bta-miR-365-3p, while these marker genes were upregulated when the expression of btamiR-365-3p was inhibited in PBMs. In contrast, overexpression of bta-miR-365-3p increased the expressions of muscle differentiation markers (MYOD1, MYOG) and promoted myoblast differentiation and myotube formation, while inhibition of bta-miR-365-3p showed the reverse effects. These observations are similar to previous results, showing that miR-365-3p promoted chondrocyte differentiation [18].

Table 2 The published functions of miR-365-3p and its validated targets

\begin{tabular}{|c|c|c|c|}
\hline Target genes & Genes name & Functions & References PMID \\
\hline HDAC4 & Histone deacetylase 4 & $\begin{array}{l}\text { Stimulate chondrocyte differentiation in chicken or } \\
\text { mouse/osteoarthritis development in human }\end{array}$ & 21856783 \\
\hline HDAC4 & Histone deacetylase 4 & Osteoarthiritis development in human & 27023516 \\
\hline TTF1 & Thyroid transcription factor1 & Regulate lung cancer & 22185756 and 26337230 \\
\hline CycD1/Bcl2 & Cyclin D1/Bcl apoptosis regulator 2 & Regulate colon cancer & 22072615 \\
\hline $\mathrm{SHC} 1$ & Src homology domain containing 1 & Gemcitabine Regulate pancreatic cancer & 24216611 \\
\hline NFIB & Nuclear factor I/B & Promote cutaneous squamous cell carcinoma & 24949940 \\
\hline CycD1/cdc25A & Cyclin D1 & Contribute to gastric tumorigenesis & 24149576 \\
\hline CycD1 & Cyclin D1 & Inhibit vascular smooth muscle cell proliferation & 24819721 and 24936138 \\
\hline Pax6 & Paired box 6 & Regulate human retinoblastoma cells & 23660406 \\
\hline Not clearly & & Transport-related stress in turkeys & 26760121 \\
\hline Kenh2 & $\begin{array}{l}\text { Potassium voltage-gated channel } \\
\text { subfamily } \mathrm{H} \text { member } 2\end{array}$ & Regulate nociceptive behaviors & 26937014 \\
\hline$\| \mathrm{L}-6$ & Interleukin-6 & Host defense & 21518763 \\
\hline $\mathrm{BCl}-2$ & $\mathrm{BCl}$ apoptosis regulator 2 & Response low-density lipoprotein stimulation & 21640710 \\
\hline
\end{tabular}




\section{Gene targets of miR-365-3p}

Several targets of miR-365-3p have been validated in other studies such as cyclin D1 (CCND1) [44, 45], histone deacetylase 4 (HDAC4) $[17,18]$, nuclear factor I/B (NFIB) [46], Pax 6 [47] thyroid transcription factor 1 (TTF1) [39], src homology domain containing 1 (SHC1) and Bax [41] (Table 2). After bioinformatics analysis, we identified that $A C V R 1$, also known as ALK2, a member of bone morphogenetic protein receptors type I, was another target of bta-miR-365-3p. As an essential member of TGF- $\beta$ family, ACVR1 has the functional roles in early embryonic development [48], lens formation [49], chondrogenesis, osteogenesis [50, 51] and cardiac hypertrophy [52]. Additionally, recurrent heterozygous mutations of $A C V R 1$ were associated with diseases in human such as fibro dysplasia ossificans progress (FOP) [53], diffuse intrinsic pontine gliomas (DIPGs) [54] and pediatric midline high-grade astrocytoma (mHGAs) [55]. The mutations of $A C V R 1$ were also associated with meat weight, eye muscle area, silverside weight, and growth traits in cattle [56, 57]. Moreover, a constitutively activating mutation of $A C V R 1$ induced the expression of Tmem176b in $\mathrm{C} 2 \mathrm{C} 12$ cells and promoted myoblast differentiation into osteoblasts [58]. In our study, we found that $A C V R 1$ is a direct target of bta-miR-365-3p, and the decreased expression of $A C V R 1$ significantly inhibited myoblast proliferation but promoted myoblast differentiation. Our study was consistent to Shi et al. research, who used the antisense oligonucleotides (AONs) to knockdown $A C V R 1$ expression in mouse, which also resulted in the induction of muscle differentiation and repression of osteoblast differentiation [59].

\section{Conclusions}

In summary, we found that bta-miR-365-3p was predominantly expressed in muscle tissues from adult and fetal stages. It also repressed the proliferation but promoted the differentiation of PBMs through the downregulation of $A C V R 1$ in cattle.

\section{Supplementary Information}

The online version contains supplementary material available at https://doi. org/10.1186/s40104-020-00528-0.

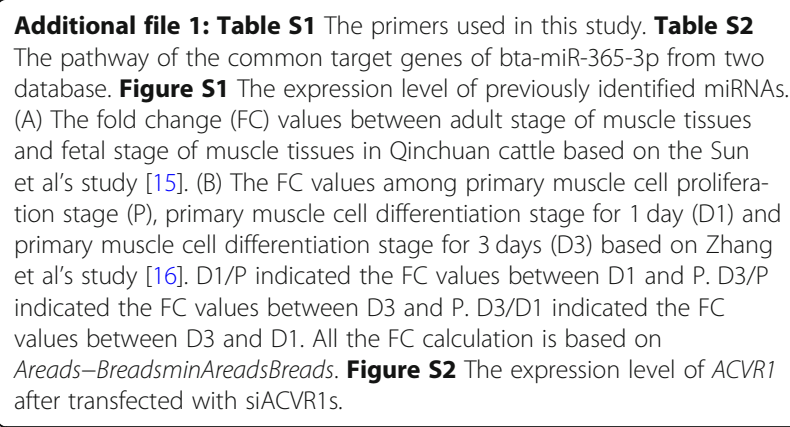

\section{Abbreviations}

ACVR1: Activin A receptor type I; CCND1: Cyclin D1; CDK2: Cyclin dependent kinase 2; DIPGs: Diffuse intrinsic pontine gliomas; DM: Differentiation medium; FOP: Sporadic fibro dysplasia ossificans progressive;

GM: Proliferation medium; GO: Gene Ontology; HDAC4: Histone deacetylase 4; KEGG: Kyoto Encyclopaedia of Genes and Genomes; MYOD1: Myogenic differentiation 1; MYOG: Myogenin; myomiR: Muscle-specific miRNAs; mHGAs: Midline high-grade astrocytoma; NFIB: Nuclear factor I/B; PCNA: Proliferating cell nuclear antigen; PBM: Primary bovine myoblast; PMSF: Phenylmethylsulfnoyl fluoride; RIPA: Radioimmunoprecipitation assay buffer; SHC1: Src homology domain containing 1; TTF1: Thyroid transcription

\section{Authors' contributions \\ DH performed major parts of the experiments. XGW and YY performed rest parts of the experiments. DH analyzed the data and drafted the manuscript. XW and BT revised the manuscript. XYL and YZH collected the animal samples. HC conceived and designed the experiments. The author(s) read and approved the final manuscript.}

\section{Funding}

This work was supported by the National Natural Science Foundation of China (No.31772574) the Program of National Beef Cattle and Yak Industrial Technology System (CARS-37). Dan Hao and Xiao Wang appreciated the scholarship from the China Scholarship Council (CSC), China.

\section{Availability of data and materials}

The data sets used and/or analyzed during the current study are available from the corresponding author on reasonable request.

\section{Ethics approval and consent to participate}

All the animal procedures were carried out according to the protocols approved by the College of Animal Science and Technology, Northwest A\&F University, China. All the experimental animals were approved by the Institutional Animal Care and Use Committee in the College of Animal Science and Technology, Northwest A\&F University, China.

\section{Consent for publication}

Not applicable.

\section{Competing interests}

The authors declare that they have no conflict of interest.

\section{Author details}

${ }^{1}$ College of Animal Science and Technology, Northwest A\&F University, Shaanxi Key Laboratory of Animal Genetics, Breeding and Reproduction, Yangling 712100, Shaanxi, China. ${ }^{2}$ Department of Molecular Biology and Genetics, Aarhus University, 8000 Aarhus C, Denmark. ${ }^{3}$ Quantitative Genomics, Bioinformatics and Computational Biology Group, Department of Applied Mathematics and Computer Science, Technical University of Denmark, Richard Petersens Plads, Building 324, 2800 Kongens Lyngby, Denmark.

Received: 12 May 2020 Accepted: 18 November 2020

Published online: 12 January 2021

\section{References}

1. Chal J, Pourquié O. Making muscle: skeletal myogenesis in vivo and in vitro. Development (Cambridge). 2017;144:2104-22.

2. Yin $\mathrm{H}$, Price $F$, Rudnicki MA. Satellite cells and the muscle stem cell niche. Physiol Rev. 2013;93:23-67.

3. Collins CA, Olsen I, Zammit PS, Heslop L, Petrie A, Partridge TA, et al. Stem cell function, self-renewal, and behavioral heterogeneity of cells from the adult muscle satellite cell niche. Cell. 2005;122:289-301.

4. Lee RC, Feinbaum RL, Ambros V. The C. elegans heterochronic gene lin-4 encodes small RNAs with antisense complementarity to lin-14. Cell. 1993;75: 843-54.

5. Li L, Xu J, Yang D, Tan X, Wang H. Computational approaches for microRNA studies: a review. Mamm Genome. 2010;21:1-12.

6. Kozomara A, Birgaoanu M, Griffiths-Jones S. MiRBase: from microRNA sequences to function. Nucleic Acids Res. 2019;47:D155-62. 
7. Braicu C, Gulei D, de Melo MB, Berindan-Neagoe I, Calin GA. miRNA Expression Assays. In: Netto GJ, Kaul KL, editors. Genomic Applications in Pathology. Cham: Springer International Publishing; 2019. pp. 51-71.

8. Huang Y, Shen XJ, Zou Q, Wang SP, Tang SM, Zhang GZ. Biological functions of microRNAs: a review. J Physiol Biochem. 2011;67:129-39.

9. Plasterk RHA. Micro RNAs in animal development. Cell. 2006;124:877-81.

10. Ge Y, Chen J. MicroRNAs in skeletal myogenesis. Cell Cycle. 2011;10:441-8.

11. Güller I, Russell AP. MicroRNAs in skeletal muscle: their role and regulation in development, disease and function. J Physiol. 2010;588:4075-87.

12. McCarthy JJ. The myomiR network in skeletal muscle plasticity. Exerc Sport Sci Rev. 2011;39:150-4

13. Jin W, Grant JR, Stothard P, Moore SS, Guan LL. Characterization of bovine miRNAs by sequencing and bioinformatics analysis. BMC Mol Biol. 2009;10:90.

14. Muroya S, Taniguchi M, Shibata M, Oe M, Ojima K, Nakajima I, et al. Profiling of differentially expressed microRNA and the bioinformatic target gene analyses in bovine fast- and slow-type muscles by massively parallel sequencing. J Anim Sci. 2013;91:90-103.

15. Sun J, Li M, Li Z, Xue J, Lan X, Zhang C, et al. Identification and profiling of conserved and novel microRNAs from Chinese Qinchuan bovine longissimus thoracis. BMC Genomics. 2013;14:42.

16. Zhang WW, Sun XF, Tong HL, Wang YH, Li SF, Yan YQ, et al. Effect of differentiation on microRNA expression in bovine skeletal muscle satellite cells by deep sequencing. Cell Mol Biol Lett. 2016;21:1-18.

17. Guan YJ, Yang X, Wei L, Chen Q. MiR-365: a mechanosensitive microRNA stimulates chondrocyte differentiation through targeting histone deacetylase 4. FASEB J. 2011;25:4457-66.

18. Yang X, Guan Y, Tian S, Wang Y, Sun K, Chen Q. Mechanical and IL-1ß responsive miR-365 contributes to osteoarthritis development by targeting histone deacetylase 4. Int J Mol Sci. 2016;17:1-15.

19. Miyake M, Takahashi H, Kitagawa E. AMPK activation by AICAR inhibits myogenic differentiation and myostatin expression in cattle. Cell Tissue Res. 2012;349:615-23

20. Agarwal V, Bell GW, Nam JW, Bartel DP. Predicting effective microRNA target sites in mammalian mRNAs. eLife. 2015;4:e05005.

21. Vejnar CE, Zdobnov EM. MiRmap: comprehensive prediction of microRNA target repression strength. Nucleic Acids Res. 2012;40:11673-83.

22. Oliveros JC. VENNY. An interactive tool for comparing lists with Venn Diagrams (2007-2015); 2018.

23. Yu G, Wang LG, Han Y, He QY. ClusterProfiler: an R package for comparing biological themes among gene clusters. OMICS A J Integr Biol. 2012;16:284-7.

24. Ekholm SV, Reed SI. Regulation of G1 cyclin-dependent kinases in the mammalian cell cycle. Curr Opin Cell Biol. 2000;12:676-84.

25. Naryzhny SN. Proliferating cell nuclear antigen: a proteomics view. Cell Mol Life Sci. 2008;65:3789-808.

26. Stacey DW. Cyclin D1 serves as a cell cycle regulatory switch in actively proliferating cells. Curr Opin Cell Biol. 2003;15:158-63.

27. Lee SH, Joo ST, Ryu YC. Skeletal muscle fiber type and myofibrillar proteins in relation to meat quality. Meat Sci. 2010;86:166-70.

28. Nie J, Liu L, Zheng W, Chen L, Wu X, Xu Y, et al. MicroRNA-365, downregulated in colon cancer, inhibits cell cycle progression and promotes apoptosis of colon cancer cells by probably targeting cyclin D1 and Bcl-2. Carcinogenesis. 2012;33:220-5.

29. Chou CH, Shrestha S, Yang CD, Chang NW, Lin YL, Liao KW, et al. MiRTarBase update 2018: a resource for experimentally validated microRNAtarget interactions. Nucleic Acids Res. 2018;46:D296-302.

30. Wang J, Song C, Cao X, Li H, Cai H, Ma Y, et al. MiR-208b regulates cell cycle and promotes skeletal muscle cell proliferation by targeting CDKN1A. J Cell Physiol. 2019;234:3720-9

31. Peng S, Song C, Li H, Cao X, Ma Y, Wang X, et al. Circular RNA SNX29 sponges miR-744 to regulate proliferation and differentiation of myoblasts by activating the Wnt5a/Ca2+ signaling pathway. Mol Ther Nucleic Acids. 2019;16:481-93.

32. Song C, Yang J, Jiang R, Yang Z, Li H, Huang Y, et al. miR-148a-3p regulates proliferation and apoptosis of bovine muscle cells by targeting KLF6. J Cell Physiol. 2019;234:15742-50

33. Sun X, Li M, Sun Y, Cai H, Lan X, Huang Y, et al. The developmenta transcriptome sequencing of bovine skeletal muscle reveals a long noncoding RNA, IncMD, promotes muscle differentiation by sponging miR125b. Biochim Biophys Acta Mol Cell Res. 1863;2016:2835-45.
34. Li H, Wei X, Yang J, Dong D, Hao D, Huang Y, et al. circFGFR4 promotes differentiation of myoblasts via binding miR-107 to relieve its inhibition of Wnt3a. Mol Ther Nucleic Acids. 2018;11:272-83.

35. Elsaeid Elnour I, Dong D, Wang X, Zhansaya T, Khan R, Jian W, et al. BtamiR-885 promotes proliferation and inhibits differentiation of myoblasts bytargeting MyoD1. J Cell Physiol. 2020; 235:1-12.

36. Hocquette JF. Endocrine and metabolic regulation of muscle growth and body composition in cattle. Animal. 2010;4:1797-809.

37. Perry RL, Rudnick MA. Molecular mechanisms regulating myogenic determination and differentiation. Front Biosci. 2000;5:d750-67.

38. Montesano A, Luzi L, Senesi P, Mazzocchi N, Terruzzi I. Resveratrol promotes myogenesis and hypertrophy in murine myoblasts. J Transl Med. 2013:11:310.

39. Qi J, Rice SJ, Salzberg AC, Runkle EA, Liao J, Zander DS, et al. MiR-365 regulates lung cancer and developmental gene thyroid transcription factor 1. Cell Cycle. 2012;11:177-86.

40. Liu Y, Zhang G, Li H, Han L, Fu A, Zhang N, et al. Serum microRNA-365 in combination with its target gene TTF-1 as a non-invasive prognostic marker for non-small cell lung cancer. Biomed Pharmacother. 2015;75: 185-90.

41. Hamada S, Masamune A, Miura S, Satoh K, Shimosegawa T. MiR-365 induces gemcitabine resistance in pancreatic cancer cells by targeting the adaptor protein $\mathrm{SHC} 1$ and pro-apoptotic regulator BAX. Cell Signal. 2014;26:179-85

42. Han JG, Jiang YD, Zhang $C H$, Yang $Y M$, Pang $D$, Song $Y N$, et al. A novel panel of serum miR-21/miR-155/miR-365 as a potential diagnostic biomarker for breast cancer. Ann Surg Treat Res. 2017:92:55-66.

43. Guo SL, Ye H, Teng Y, Wang YL, Yang G, Bin LX, et al. Akt-p53-miR-365cyclin D1/cdc25A axis contributes to gastric tumorigenesis induced by PTEN deficiency. Nature communications. Nat Publ Group. 2013:4:1-11.

44. Zhang P, Zheng C, Ye H, Teng Y, Zheng B, Yang X, et al. MicroRNA-365 inhibits vascular smooth muscle cell proliferation through targeting cyclin D1. Int J Med Sci. 2014;11:765-70.

45. Kim MH, Ham O, Lee SY, Choi E, Lee CY, Park JH, et al. MicroRNA-365 inhibits the proliferation of vascular smooth muscle cells by targeting cyclin D1. J Cell Biochem. 2014;115:1752-61.

46. Zhou M, Zhou L, Zheng L, Guo L, Wang Y, Liu H, et al. miR-365 promotes cutaneous squamous cell carcinoma (CSCC) through targeting Nuclear Factor I/B (NFIB). Plos One. 2014;9:e100620.

47. Wang J, Wang X, Wu G, Hou D, Hu Q. MiR-365b-3p, down-regulated in retinoblastoma, regulates cell cycle progression and apoptosis of human retinoblastoma cells by targeting PAX6. FEBS Lett. 2013;587: 1779-86

48. Gu Z, Reynolds EM, Song J, Lei H, Feijen A, Yu L, et al. The type I serine/ threonine kinase receptor ActRIA (ALK2) is required for gastrulation of the mouse embryo. Development (Cambridge). 1999;126:2551-61.

49. Rajagopal R, Huang J, Dattilo LK, Kaartinen V, Mishina Y, Deng CX, et al. The type I BMP receptors, Bmpr1a and Acvr1, activate multiple signaling pathways to regulate lens formation. Dev Biol. 2009:335:305-16.

50. Lin S, Svoboda KKH, Feng JQ, Jiang X. The biological function of type i receptors of bone morphogenetic protein in bone. Bone Res. 2016;4 16005.

51. Zhang D, Schwarz EM, Rosier RN, Zuscik MJ, Puzas JE, O'Keefe RJ. ALK2 functions as a BMP type I receptor and induces Indian hedgehog in chondrocytes during skeletal development. J Bone Miner Res. 2003;18:1593-604

52. Shahid M, Spagnolli E, Ernande L, Thoonen R, Kolodziej SA, Leyton PA, et al. BMP type I receptor ALK2 is required for angiotensin II-induced cardiac hypertrophy. Am J Physiol Heart Circ Physiol. 2016;310:H984-94.

53. Shore EM, Xu M, Feldman GJ, Fenstermacher DA, Brown MA, Kaplan FS. A recurrent mutation in the BMP type I receptor ACVR1 causes inherited and sporadic fibrodysplasia ossificans progressiva. Nat Genet. 2006;38:525-7.

54. Taylor KR, Mackay A, Truffaux N, Butterfield YS, Morozova O, Philippe C, et al Recurrent activating ACVR1 mutations in diffuse intrinsic pontine glioma. Nat Genet. 2014;46:457-61

55. Fontebasso AM, Papillon-Cavanagh S, Schwartzentruber J, Nikbakht $\mathrm{H}$, Gerges N, Fiset PO, et al. Recurrent somatic mutations in ACVR1 in pediatric midline high-grade astrocytoma. Nat Genet. 2014;46:462-6.

56. Cheng J, Cao X, Hao D, Ma Y, Qi X, Chaogetu B, et al. The ACVR1 gene is significantly associated with growth traits in Chinese beef cattle. Livest Sci. 2019;229:210-5. 
57. Novianti I. Molecular genetics of cattle muscularity; 2011.

58. Yano M, Kawao N, Tamura Y, Okada K, Kaji H. A novel factor, Tmem176b, induced by activin-like kinase 2 signal promotes the differentiation of myoblasts into osteoblasts. Exp Clin Endocrinol Diabetes. 2014;122:7-14.

59. Shi ST, Cai J, de Gorter DJJ, Sanchez-Duffhues G, Kemaladewi DU, Hoogaars WMH, et al. Antisense-oligonucleotide mediated exon skipping in activinreceptor-like kinase 2: inhibiting the receptor that is overactive in fibrodysplasia ossificans progressiva. Plos One. 2013;8:e69096.

Ready to submit your research? Choose BMC and benefit from:

- fast, convenient online submission

- thorough peer review by experienced researchers in your field

- rapid publication on acceptance

- support for research data, including large and complex data types

- gold Open Access which fosters wider collaboration and increased citations

- maximum visibility for your research: over $100 \mathrm{M}$ website views per year

At $\mathrm{BMC}$, research is always in progress.

Learn more biomedcentral.com/submissions 